\title{
GENE EXPRESSION
}

\section{Transcripts from a spliceosome}

Two new methods provide high-resolution profiles of splicing events in yeast.

R NA splicing is a hallmark of eukaryotic life, the handiwork of a highly regulated structure called the spliceosome. The term "spliceosome" actually refers to a series of complexes that gain and lose protein parts as pre-mRNA is bound, the complex is activated, and two serial transesterifications remove an inter-exon region in a manner resembling a cinematic film edit. Profiling the full range of splicing intermediates is not a straightforward task, and has had to wait for two recent approaches.

With his colleagues, Hiten Madhani at the University of California, San Francisco, affinity-purified Flag-tagged Prp19, a key component of active spliceosomes, and then carried out $3^{\prime}$-end profiling of associated RNA using native elongating transcript sequencing (to identify cleaved $5^{\prime}$ exons and excised introns) and total RNA-seq.
The researchers applied the approach to three common yeast species and compared the results with those of standard mRNA-seq to quantify splicing precursors, intermediates and products. The method detected rare noncanonical events, and Bayesian modeling of the data identified intron features associated with spliceosome disassembly during stalled splicing.

In related work, the team of Melissa Moore of the University of Massachusetts Medical School purified activated spliceosomes by using a triple-tagged fission yeast strain that they had developed previously. From this, they generated one sequencing library to profile all associated RNA, a second library to map intron $5^{\prime}$ ends, and a $3^{\prime}$-hydroxy library to obtain high-confidence splice sites. Their work improved intron annotations, and additional experiments using micrococcal nuclease mapped RNA footprints representing stable spliceosome interactions.

Much as ribosome profiling has been a boon for probing transcripts undergoing translation, the establishment of these new spliceosome profiling methods will enable deeper analyses of splicing regulation.

\section{Tal Nawy}

Published online: 2 July 2018

https://doi.org/10.1038/s41592-018-0058-3

Research papers

Burke, J. E. et al. Spliceosome profiling visualizes operations of a dynamic RNP at nucleotide resolution. Cell 173, 1014-1030 (2008).

Chen, W. et al. Transcriptome-wide interrogation of the functional intronome by spliceosome profiling. Cell 173, 1031-1044 (2018).

\section{We Create Solutions}

Ultra Precise Motion Control - D.C. Servo motors down to $20 \mathrm{~nm}$, piezos down to $1 \mathrm{~nm}$, and low drift XYZ stages.

Microscopy - Automation, modular microscopes, autofocus complete light sheet systems, and components.

OEM - Custom designed systems to user specifications.

www.asiimaging.com

info@asiimaging.com

(800) 706-2284 or (541) 461-8181

VISIT US AT:

- The $3^{\text {rd }}$ EMBO Practical Course on Light Sheet Microscopy - Aug. $2^{\text {nd }}-11^{\text {th }} \cdot$ Dresden, Germany

-The $10^{\text {th }}$ Annual LSFM Conference $\cdot$ Aug. $12^{\text {th }}-15^{\text {th }} \cdot$ Dresden, Germany

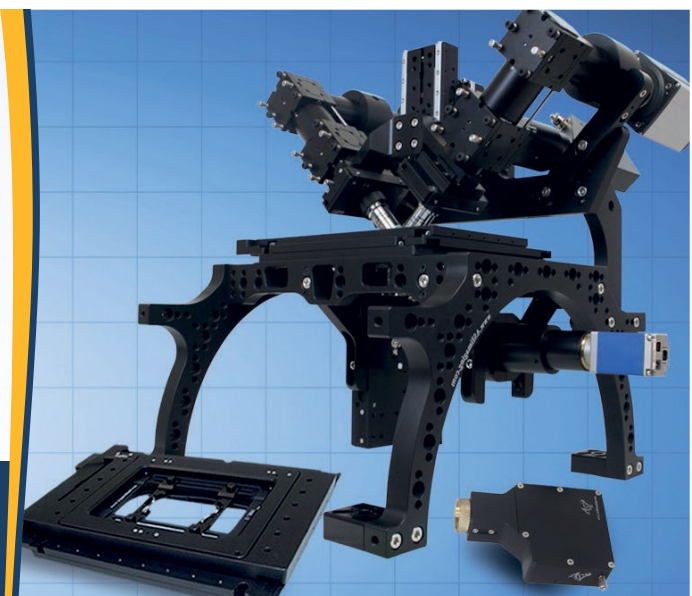

